\title{
Preliminary project design for insect production: part 2 - organism kinetics, system dynamics and the role of modelling \& simulation
}

\author{
R. Kok \\ Bioresource Engineering, Macdonald Campus of McGill University, 21,111 Lakeshore Rd, Ste-Anne-de-Bellevue, QC H9X \\ 3V9, Canada; robert.kok@mcgill.ca
}

Received: 13 November 2020 / Accepted: 30 November 2020

(c) 2021 Wageningen Academic Publishers

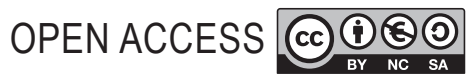

\section{TECHNICAL REPORT}

\begin{abstract}
During preliminary project design (PPD) an entopreneur can investigate a variety of process types, organism/feed combinations, operating conditions and procedures, control approaches, etc. before deciding on a specific system arrangement and proceeding to a more formal design stage. With modelling and simulation the effort required to locate a high-value point within the overall possibility space for a system can be greatly reduced because much of the development work can be carried out in a virtual environment. Nevertheless, to formulate models of the organism/feed kinetics as well as other system aspects, this approach must be based on experimental data. And, of course, results of a simulation study must be verified empirically. A simulation-based approach to PPD allows an entopreneur to study the dynamics of a wide variety of system arrangements and to gain insight into how a given arrangement is likely to perform with different parameter values and disturbances.
\end{abstract}

Keywords: kinetics, dynamics, modelling, simulation

\section{Introduction}

The overall purpose of preliminary project design (PPD) is to facilitate an entrepreneur's exploration of the conceptual possibility space for a proposed project and to help them make some preliminary decisions about that project. In essence, PPD is the activity that links early concept development, initial data acquisition and project definition to formal engineering design, construction, and ultimate plant operation (Kok, 2021a; Figure 1). Although the principles of PPD are applicable to pretty well all types of projects, the discussion here is oriented to the mass production of insects on a commercial scale, and more specifically, the rearing of black-soldier fly larvae, mealworms and other, similar kinds of organisms. Often, even before a project's PPD phase is started, some preliminary decisions will have been made about, e.g. the organism and feed, approximate scale of operation, process type, target market sector, etc. However, during PPD these aspects may be adjusted considerably, or even changed altogether. Thus, PPD is about evaluating the potential of a spectrum of options in order to obtain a fair understanding of the pros and cons of each and to select those options which best fit the entopreneur's intent and capabilities. In essence, PPD is a structured approach to decision making before any major, formal commitment is made to a project.

This paper is the second (Part 2) in a set of four. In the first paper (Part 1: Kok, 2021a) the overall mass and energy/ heat balances of a rudimentary larval rearing setup were explored by means of a simple calculator model. The model was presented in spreadsheet form and is available to the reader as electronic supplementary material for use with their own data. Generally, in considering a production project, this will probably be the first item to address since it will yield some basic, if rudimentary, information about quantitative aspects. Then, based on this, an initial likelihood of a proposed project's financial success can be estimated. Evidently, if this likelihood is too far below an investor's hopes and expectations the project objectives and scope will need to be re-examined. In short, a calculator model of this type allows an investor and entopreneur to fairly quickly evaluate the potential of a proposed project while also providing them with the opportunity to 


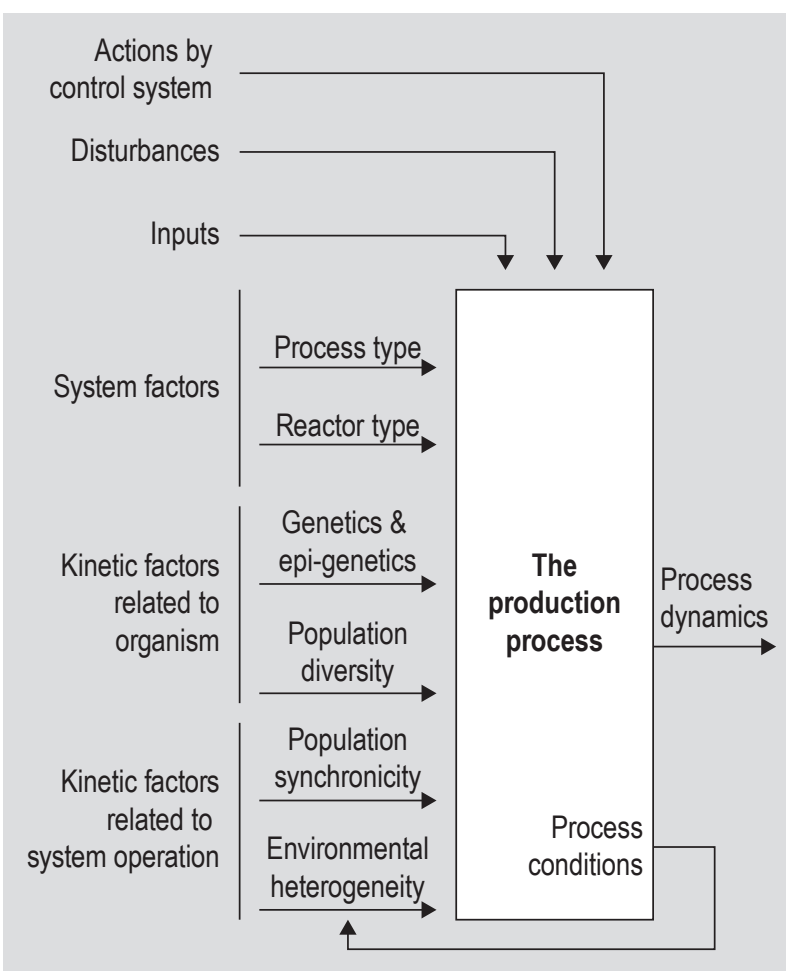

Figure 1. The relationships between influences on process dynamics.

investigate related alternatives, e.g. operation with a variety of different feeds, etc. In essence, the calculator model provides a framework for the entopreneur to work in and, at the same time, lets them know what data is required to make a rational decision. If adequate data cannot be obtained for an alternative, it is immediately clear that the reliability of the predictions is fairly low. One advantage of a simple calculator model of this type is that the results are available almost instantaneously so that the sensitivity of any of the outputs to variations in the inputs can be quickly established. Overall, the intent of providing the model is to facilitate the entopreneur and investor in making some preliminary decisions for a project about production volume, organism, feed, etc. on a rational basis.

After some of these preliminary decisions have been made, the type of process that will be employed and the type of reactor the process will be based on need to be considered. The choices related to these two items will be strongly influenced by a host of factors including the scope of the project, the target organism, technical capacity available, personal preferences, etc. A major item in these considerations is how the process will perform dynamically, i.e. how the system state and output will vary with time. Thus, whereas in Part 1 (Kok, 2021a) the overall process input/output balance was of primary concern, in this paper process dynamics are considered and, specifically, how they can be dealt with, thought about and anticipated.
Three main types of influences on the nature of the process dynamics are considered here: system factors, kinetic factors and control system actions. Of course, process inputs and disturbances will affect the specifics of the dynamics as well, but not the character of the system's response. The relationships between the factors are illustrated in Figure 1. They were also briefly discussed by Kok (2017). Two of the kinetic factors are directly related to the inherent properties of the organism while the other two are related to system operation. One of the latter two, environmental heterogeneity, is directly influenced by the process conditions which are the result of all the factors combined. Below, the interaction between the factors is examined with a stress on how a modelling and simulation approach can be used to understand and, at times, exploit the relationships between them. It should be noted that, as explained further below, 'modelling and simulation' here refers to a very general set of activities, including purely mental ones.

In Part 3 (Kok, 2021b) of this set a number of process types suitable for insect mass production at various scales are then explored in terms of their input/output dynamics, ease of process operation, equipment complexity, labour requirements, suitability for automation, etc. Finally, in light of these former aspects, in Part 4 (Kok, 2021c) overall process dynamics as the convoluted result of choices about the organism/feed combination and process type are discussed, together with some considerations of the physical facilities required to house the project. Altogether, the material is structured to help entopreneurs deal with the PPD of projects ranging from the very small, manuallyoperated enterprise to the full-scale, automated industrial plant.

\section{Modelling and simulation}

During PPD one normally reasons about a project and the process on which it is based according to some type of simulation. In its makeup such a simulation may be situated anywhere between purely informal and formal. If the simulation of a project is entirely informal it will probably consist of purely mental considerations of a number of possible scenarios and the, again purely mental, evaluation of their foreseen likelihood of success. Under such conditions the criteria for success may never be explicitly stated and the final judgment may be made purely intuitively. Whereas this approach has been quite successful in many instances (some people having 'good insight' and 'talent'), it can be difficult to justify judgments and decisions, especially since the reasoning chain followed in intuitive decision-making is usually not accessible to even the individual themselves. Although this simulation method can be quite sophisticated (after all, the human brain is still the most advanced computer we have available), without the presentation of a clear and explicit reasoning chain at the 
conclusion it can be difficult to convince potential investors to underwrite a project. As an alternative to the purely informal approach, a number of semi-formal simulation methods can be followed in which 'insight' and 'hunches', etc. can be supported with calculations and references to other, similar projects that have already proven successful. Then, at the far end of the spectrum lies the truly formal simulation. This is grounded in computer-based reasoning combined with evaluation of all options on the basis of clearly stated criteria so that the entire reasoning chain and all intermediate reasoning steps and results are available for inspection. Thus, with a formal simulation approach it is much easier to explain and justify the decisions that are made and to discuss the advantages and disadvantages of various options with other parties. The disadvantage of the formal approach is that the reasoning followed is only as sophisticated as the programming of the simulation (and the model on which it is based). Realistically, a modern, potential entopreneur will probably base their reasoning partly on a formal simulation approach and combine the outcome of that with the results from one or more semiformal and informal methods so as to arrive at an ultimate decision about whether to proceed and try to assemble the means to market the project. After all, the concept will have to be promoted to potential investors who will be convinced by the entopreneur's passion and enthusiasm for the project just as much as by rational argument and cold facts about profitability. Only the formal simulation approach is dealt with here.

Any simulation of a process will be based on a model of that process and, in turn, the model will be based on specific data about the system of interest, as well as more general knowledge. For a PPD exercise, really only a preliminary step in project development, the process model may be relatively coarse and incomplete and be based on simplifying assumptions in order to expedite the procedure. It should, however, be detailed enough to yield a 'fair impression' of process performance in the region of interest of the conceptual possibility space. Thus, part of the 'art' of PPD is choosing which aspects to model to what extent and how to interpret the results of the simulations and the ensuing reasoning based upon them. During a PPD exercise it will frequently occur that it becomes desirable to consider an option for which no data is available. For example, the system model may be based on larvae growing on a feed with soy meal as protein source. If it then seems interesting to partially replace that ingredient with cottonseed meal, more data will be needed to expand the range of that model. Thus, in this instance, PPD considerations lead to a data requirement. In another situation a simulation may suggest that system performance can be improved by varying the temperature during larval rearing. That would then need to be verified experimentally, leading to more data requirements, reasoning, etc. Therefore, in the approach presented here model-based reasoning and experimental exploration and verification are seen as the twin components of an iterative knowledge engine that one can use to explore the possibility space for a project during PPD. Generally, the overall objective is to identify a family of process operating conditions that will give rise to process stability as well as relatively high values for an objective function such as financial reward.

When the model of a process is made up only of simple algebraic relationships between independent variables (such as feed protein content) and dependent ones (such as productivity per unit reactor volume) there is essentially no difference between the simulation and the model on which it is based. This was the case in Part 1 of this set (Kok, 2021a) in which the computation of the overall mass and energy/heat balances of a single tray of larvae growing on a bed of feed was presented. But when the model is made up of more complex elements, some of which may be relationships between variables that are time based such as differential equations, the model and the simulation are quite different entities. Now the simulation is more like an exoskeleton within which the model is contained and integrated with respect to time. The structure of the modelsimulation system is illustrated in Figure 2. As shown, some types of elements that may be included in a complex model are algebraic and differential equations as well as data, rules and cases respectively held in databases, rulebases and casebases, and even stories.

With simulation based on a dynamic system model timevarying inputs (and system disturbances) can be taken into account, as well as time-varying responses so that, in turn, dynamic, time-varying system states and outputs can be computed. This type of knowledge is very important because there can be very significant variations in process variables during, e.g. the rearing of a batch of larvae. Concomitantly, the wave form and magnitude of such variations are often strongly affected by the process type and the processing conditions. This is discussed in more detail in the sections below.

As mentioned above, a spectrum of simulation methods is available, ranging from the informal to the strictly formal, with any formal method being based on a computational approach. Evidently, a large number of languages, packages and simulation systems are available with which a model can be written and used in a simulation. It is the author's opinion, however, that for the modelling and simulation of a biological production system the use of an objectbased approach provides for maximum inclusiveness and flexibility (Parrott and Kok, 2001a,b, 2002). Although this does require some investment it has the advantage that it readily allows for minor model adjustments as well as major modifications. Thus, a model (within a simulation) of this type can function as a pivotal instrument in the PPD exercise by facilitating a wide-ranging exploration 


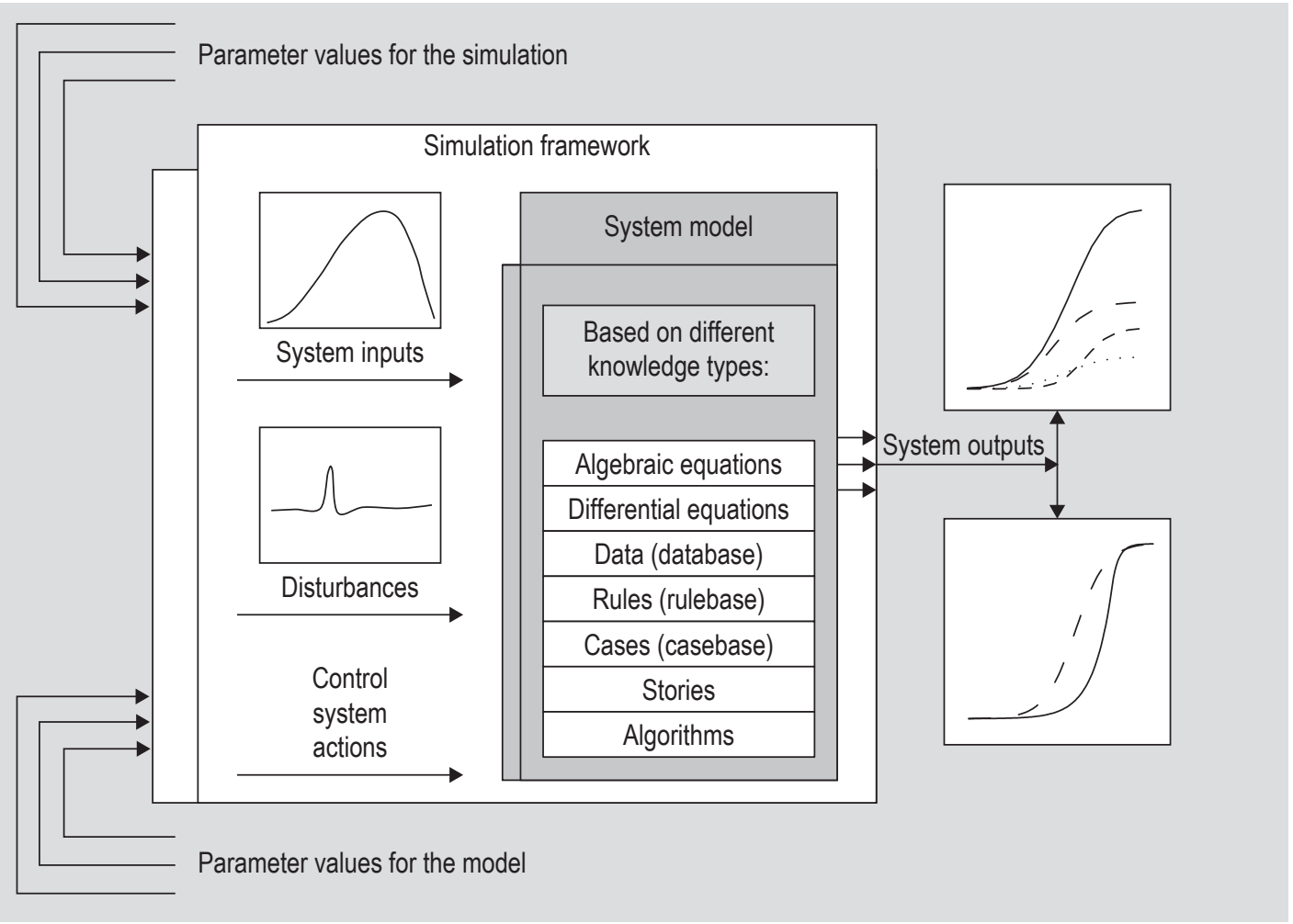

Figure 2. The relationship between the simulation framework and the dynamic system model.

of the possibility space, both for the process and for the control approach.

Although a formal simulation exercise as part of PPD comes at a cost, it also delivers major benefits. Clearly, those benefits come to light most eminently when the model reflects reality to a good degree, illustrating the fact that any computational effort is only one of the twin components of this type of knowledge engine, the second one being data and knowledge acquisition about the system under investigation.

\section{Kinetic factors}

In chemistry the term 'kinetics' refers to the rate of a reaction between molecules, that rate usually depending on conditions such as temperature, the concentrations of various reactants, the presence of a catalyst, etc. 'Kinetics' is also commonly used in reference to the growth of microorganisms like bacteria and yeasts. The mathematical treatment of such growth is well established, especially for production in submerged culture (Aiba et al., 1973). Accordingly, it is used here in the same sense to refer to the reaction rate between a macroorganism such as an insect and its nutrients, subject to the environmental conditions of the situation. As is true for chemical and microbiological systems, the kinetics of an entotechnological system can vary substantially with the parameters of a process (e.g. Diener et al., 2011; Nyakeri et al., 2017) so that the details for each system and each set of circumstances must be determined experimentally.

The first kinetic factor considered here is related to genetics and epi-genetics which determine the organism's response to a given history of environmental conditions. Models of various complexity can be constructed of this response, with different variables being included or excluded. Thus, although it is mostly the organism growth rate that is dealt with in the discussion below, a comprehensive kinetic model will actually consist of a functional description of how the entire reaction will proceed. Hence, if such a model is reasonably complete, besides being a description of how a given quantity of eggs or young larvae will grow and how the composition of the population will change during its development, it will also be a functional description of the rates of substrate disappearance, oxygen consumption, carbon dioxide and heat production, the generation of various types of larval waste, etc. Also, there is a large spectrum of situations for which different kinetic models might be developed in accordance with how the environmental conditions will be varying during the rearing period. At the one end of the spectrum the control system is able to regulate the environmental conditions so they remain entirely stable throughout the rearing period. The general equations for this type of kinetic model are then as follows:

$\mathrm{dX} / \mathrm{dt}=\mathrm{F} 1 \mathrm{a}\{\mathrm{X}, \mathrm{Z}, \mathrm{A} 1\}$ 
$\mathrm{dY} / \mathrm{dt}=\mathrm{F} 1 \mathrm{~b}\{\mathrm{X}, \mathrm{Z}, \mathrm{A} 2\}$

where:

A1, A2 = vectors of kinetic parameters

$\mathrm{t}=$ time

$\mathrm{X}=$ vector of larva-related variables including: larval weight and development; larval composition such as protein, fat, etc.

$\mathrm{Y}=$ vector of time-varying variables including: feed consumed; oxygen consumed; carbon dioxide given off; latent heat given off as evaporated water; sensible heat given off; waste materials produced; and water consumed. $\mathrm{Z}$ = vector of non-time-varying variables including: feed type; feed availability; temperature; water availability; oxygen availability; and carbon dioxide presence.

In Figure 3 are depicted three component synthesis rate curves (protein, fat, and other materials) corresponding to a hypothetical kinetic model as described by Equation 1a. The curves are for an individual larva; the three rates were also integrated over a 14-day period and added, resulting in the larval development curve (dry weight vs time) culminating in a final dry weight of $120 \mathrm{mg}$.

At the other end of the spectrum, the environmental history in the reactor is allowed to evolve freely and all variables allowed to find their own levels without interference of the control system. Larval growth may, for instance, generate heat and humidity in the bed of substrate on which the larvae are being reared, causing the environmental conditions during the rearing history to be time-varying to a greater or lesser degree. This will lead to a substantially more complicated situation, the general equations for this type of kinetic model being:

$\mathrm{dX} / \mathrm{dt}=\mathrm{F} 2 \mathrm{a}\{\mathrm{X}, \mathrm{Y}, \mathrm{B} 1\}$

$\mathrm{dY} / \mathrm{dt}=\mathrm{F} 2 \mathrm{~b}\{\mathrm{X}, \mathrm{Y}, \mathrm{B} 2\}$

where:

$\mathrm{B} 1, \mathrm{~B} 2$ = vectors of kinetic parameters

$\mathrm{t}=$ time

$\mathrm{X}$ = vector of larva-related variables including: larval weight and development; and larval composition such as protein, fat, etc.

$\mathrm{Y}=$ vector of time-varying variables including: feed type; feed availability; feed consumed; temperature; water availability; water consumed; oxygen availability; oxygen consumed; carbon dioxide presence; carbon dioxide given off; latent heat given off as evaporated water; sensible heat given off; and waste materials produced.

Clearly, there are models that can be developed for situations intermediate to these two extremes. For example, the environmental conditions might be adjusted once or twice during the rearing history, but otherwise held steady by the control system. Evidently, as the rearing situation becomes more complicated and variable more experimental effort

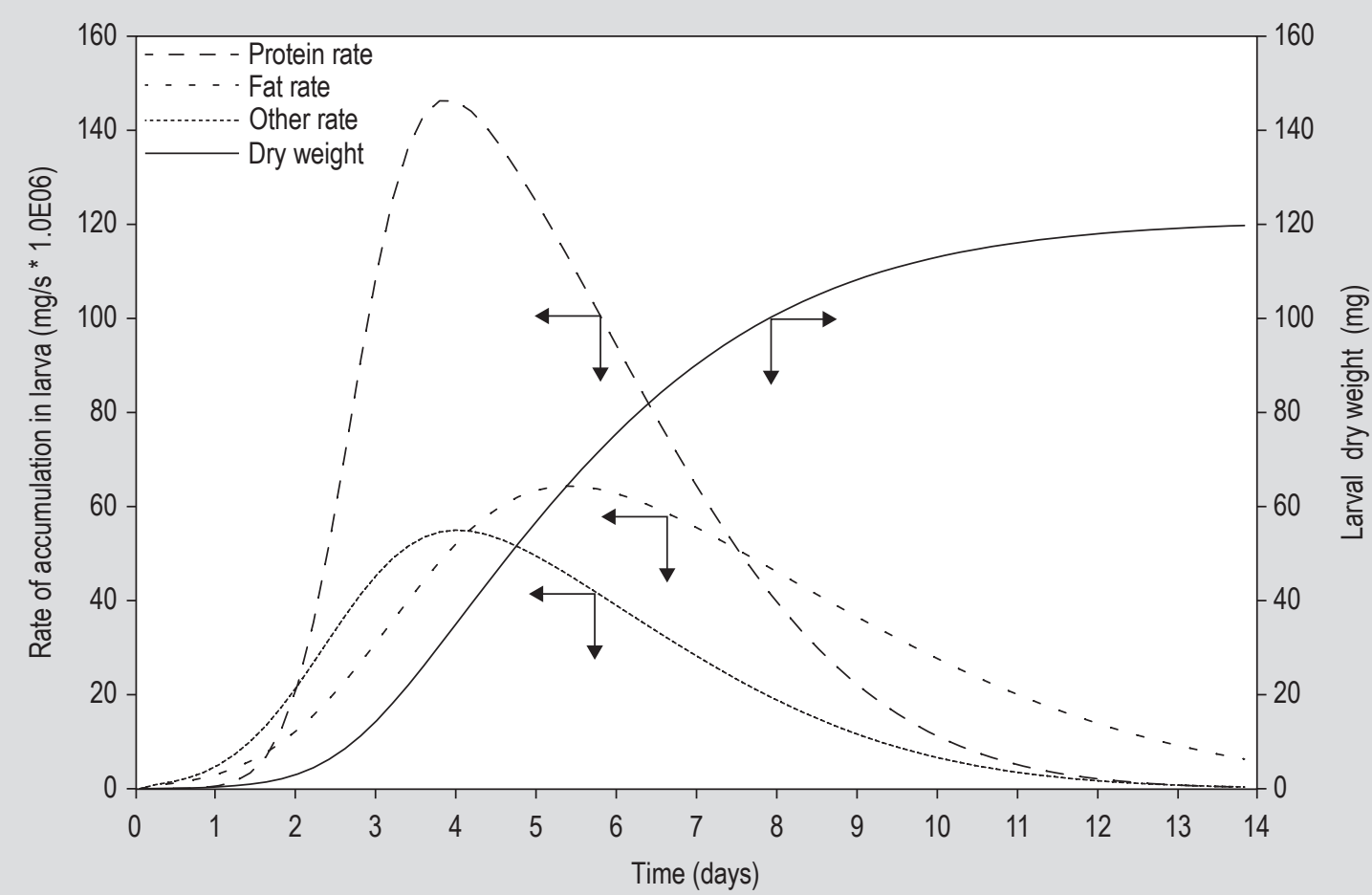

Figure 3. Component synthesis rates and the development curve for a single larva. 
(or a very good literature source) will be required to obtain sufficient information to formulate an appropriate kinetic model. Regardless of the type of model, the information about the kinetic relationships described by these equations may be ultimately made available to a simulation framework in either analytical or numerical format.

In the models described above the first organism-related factor, genetics and epi-genetics, is dealt with, but not the second which is population diversity. Thus, referring to Equation set 1 and Figure 3, one would expect that if a thousand perfectly synchronised eggs (i.e. all exactly the same age) are subjected to the same environmental history (no heterogeneity), the same curves would be obtained, amplified a thousand times, the population attaining a total dry weight of $120 \mathrm{~g}$ after 14 days. As is true for pretty well all biological populations, however, the larval population is likely to be multifarious to a some degree in their genetic and epigenetic characteristics and this will result in diversity in their responses to the environmental history to which they are subjected. With other words, the rate curves for the individuals will not be exactly the same even if the larvae are subjected to the same histories so that the larvae will be distributed to some extent in their degree of development at the end of the 14-day incubation period. This is shown as Case 1 in Figure 4. Here the final development distribution is Gaussian in shape but that is for convenience of illustration only; the organisms' characteristics could have any distribution which would be reflected in the end result for the population. To take the population's genetic diversity into account in a simulation of the process it will need to be reflected in the organism kinetic model. This is best done by structuring some of the parameters in vectors $A$ and $B$ in equation sets 1 and 2 as probability functions. The shape of these functions would, of course, need to be determined experimentally.

Whereas the first two kinetic factors are directly related to the genetics of the population, the other two are of a more operational character. The third factor is related to it being quite unlikely to obtain a population of eggs that are exactly the same age. On the contrary, under real conditions it is much more likely that the eggs in a batch will be distributed in their ages. If this non-synchronicity of the eggs is taken into account at the same time as the organism's genetic diversity, the development distribution

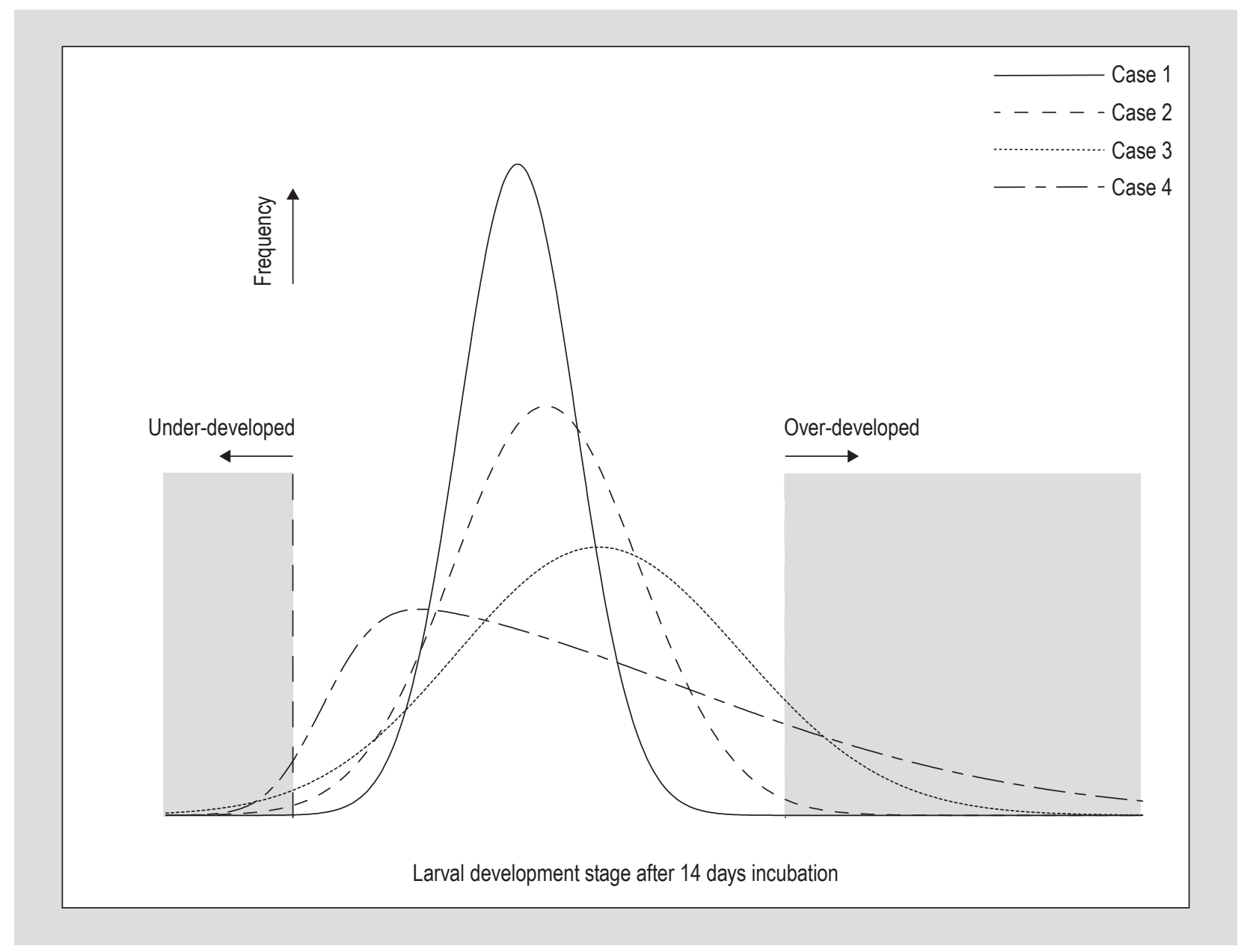

Figure 4. Development distributions for a larval population. 
of the larvae at the end of the incubation period will be wider than when only the diversity is considered. As well, the shape of the distribution will probably be affected. This is shown as Case 2 in Figure 4, for which the development distribution is a slightly skewed normal curve. ${ }^{1}$ As shown in Figure 4, it is likely that the widening of the development curve will lead to higher reject rates caused by both underdeveloped and over-developed larvae.

Whereas the third kinetic factor is related to the age distribution of the eggs being supplied to the process, the fourth is related to individuals being exposed to somewhat different environmental histories during their residence time in the reactor. Thus, even though a batch may be reared in the same tray or bin or on the same belt, at various times the organisms may well be subjected to quite different environmental conditions causing their environmental histories to not be the same. For example, a larva may spend its early life near the edge of a tray where it may be slightly cooler and drier than in the centre and that will affect its growth curve in accordance with its individual genetic and epigenetic characteristics. This applies to all larvae in the population so that the shape of the final development distribution will be affected in some way by heterogeneity in environmental conditions. In Figure 4 this is illustrated with Cases 3 and 4, for which the distributions are quite skewed, one somewhat more extreme than the other. Due to the greater spread of these development distributions as compared to Case 2, the reject rates due to larval under-development and over-development are again higher than before. As for the other kinetic factors, it is likely the environmental heterogeneity will be distributed in some way and the parameters for the distributions of the variables involved will need to be supplied to the simulation as part of the process model.

A key point to consider here is that the cumulative effect of the three causal distributions (diversity, nonsynchronicity and environmental heterogeneity) is not merely additive. Instead, the effects will be interactive and will be convoluted. ${ }^{2}$ A very significant feature of this phenomenon is that, although the three causal distributions may be fairly narrow in themselves, due to convolution the final distribution may become surprisingly wide and quite skewed, thus resulting in higher than anticipated reject rates from the final population, both as underdeveloped and over-developed individuals. Developing a good understanding of how these causal factors interact and how their distributions are shaped and convoluted

\footnotetext{
${ }^{1}$ Note that this curve is hypothetical only and serves merely as an illustration for the discussion.

${ }^{2}$ For a good discussion of convolution see https://en.wikipedia.org/ wiki/convolution.
}

while the larvae pass through the reactor is crucial for making decisions about a number of aspects of the process, ultimate process type choice and its design, as well as the structure of the control system. Manifestly, it is preferable to develop such insight during the earlier phases of a project's development since it can be useful when searching a possibility space for 'low-hanging fruit' type of opportunities to improve process performance and productivity.

\section{The system model, simulation and process dynamics}

'Process dynamics' refers here to the assembly of the histories of all process variables. Typically, for a bioproduction process such as the rearing of an insect it is the result of the growth environment acting on the organism within the rearing reactor and associated equipment. The dynamics of the process come about as the interaction and convolution of the processing approach, the processing conditions and the kinetic factors of the organism. Studying these dynamics by means of simulation during the PPD phase of project development allows one to predict how a given configuration of a proposed system will perform and thus allows for discussion and planning well before any physical implementation takes place. A system model and simulation can also be very useful later on during project development to study how system performance might be modified and improved, etc.

As illustrated in Figures 1 and 2, in general, the rearing system relies for its functioning on its inputs but is subject to disturbances while being governed by a control system. This means that models of these various system components, including the inputs, must be made available to the simulation in order to predict how it will perform and respond dynamically to any disturbance. It does not mean, however, that these models need be very detailed or complicated, especially at the start of a project discussion. Thus, one might begin a simulation study for a proposed project with quite rudimentary models of all the system components and then gradually increase their sophistication as the need arises, in this way gradually developing the models as the study progresses. This procedure of gradual model development is discussed in more detail in the section below on the applications of simulation. Regardless of how coarse or how detailed the models are, in order to be reasonably representational, the components that will need to be supplied for the simulation are as illustrated in Figure 5.

As shown, besides the model of the rearing system, there will be ancillary models of the disturbances and the control system as well as of the inputs. The latter must encompass all materials and services being supplied to the system such as eggs, feed, water, air, energy being supplied, cooling, 


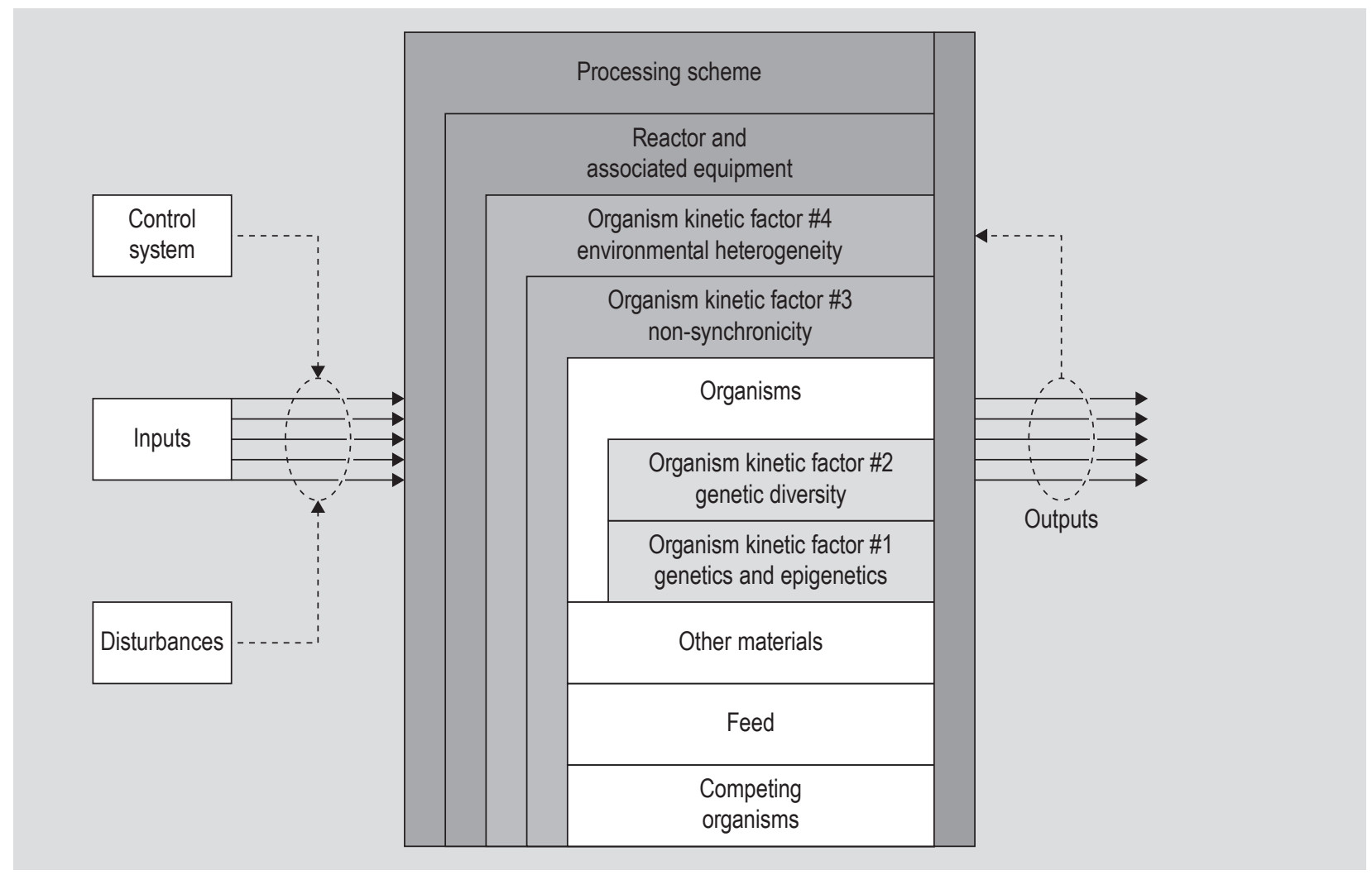

Figure 5. Components of a system model.

etc., including the states of intensive properties such as the temperatures and enthalpies of these. The same applies to the outputs. In this scheme the environmental histories to which the organisms are subjected and how they respond to these are determined by a combination of how the process is operated, how the reactor and its associated equipment is arranged, the organism kinetics, how the inputs and reactor conditions are manipulated by the control system, how some of the outputs can or will affect the environmental histories, and how disturbances will impact the production system. 'Other materials' refers to substances such as larval waste described in Part 1 (Kok, 2021a).

As mentioned, the system model components (and the ancillary models) can be as complicated or as simple or as desired. For instance, at the start of a simulation study one might simply not take into account any possibility of disturbances, set all controls to nil, assume stable inputs, define a simple processing scheme and reactor, ignore any environmental heterogeneity and any feedback from the outputs, and disregard any non-synchronicity, genetic diversity as well as the possible presence of competing organisms such as bacteria. In this case the simulation output will be a straightforward history of a uniform population growing under unrestrained conditions, the result mainly depending on the organism's genetics under the conditions dictated by the processing scheme and the equipment. The histories of organism development, feed depletion, other material accumulation, temperature, enthalpy of the outflowing air, etc. will then simply unfold in a natural, unbridled manner.

Then, as the simulation study progresses one can add elements to the models of the various system components in order to make it reflect reality better and, hopefully, elicit a dynamic response that matches. For example, one might wish to not ignore the presence of bacteria that will compete for feed with the larvae. To do so, a sub-model of bacterial kinetics will need to be added to the overall system model. This can be useful to examine how some of the inputs might be manipulated in order to reduce the "bacterial composting effect.' This would also require the inclusion of a control module to carry out such manipulation.

In the relatively simple examples above the models are of the 'lumped-parameter' type meaning that the entire population of organisms is dealt with as a single entity, the same being true for the entire population of bacteria. If, however, genetic diversity, non-synchronicity and heterogeneity of the environmental conditions are to be taken into account, the system model components need to be more complex and of a distributed parameter type. As well, the processing scheme may be operated and the reactor set up to deal simultaneously with multiple, timespaced batches of organisms (this is discussed in detail in Part 3 of this set; Kok, 2021b) so that this possibility will 
need to be accommodated in the model too. Although it is not always necessary to include all the above-mentioned types of variability and multiplicity in a system model, generally, one would include at least several of them and the modelling approach should reflect that. As pointed out, in the author's opinion, the object-based approach is very suitable for writing such models. It readily allows for a population or a phenomenon to be divided into groups at any resolution (even down to individuals), reflecting the distribution of the characteristic of interest. For example, a population of 100,000 eggs whose age distribution is known might be divided into a thousand objects whose ages have the same distribution as the population. At the same time, although in reality the environmental heterogeneity will be distributed continuously, it might be approximated with a hundred zones in which conditions are constant so that the thousand slightly different organism objects will variously experience somewhat different histories in a manner similar to that would happen in a physical reactor. This approach can be expanded to include any number of distributed phenomena in a model and is a simple and easy-to-understand way to approximate the convolution of a number of distributed phenomena. Thus, all in all it is a relatively easy way to model any number of trays or bins or groups of such to pass through the system while small, lumped-parameter batches of organisms are followed in detail as they are subjected to various inputs, environmental conditions, disturbances, etc. If necessary, this also allows for direct interaction between model elements, e.g. between organisms and the bacterial population. The overall progress of the process as well as inputs, outputs, etc. are then computed at the end of each simulation time step by adding the contributions of the small batches of larvae and competing organisms. All system mass and energy accounting also happens at that stage, with data being passed on to the simulation framework for bookkeeping and logging. At the end a complete and detailed picture of the system dynamics, including the histories of the mass and energy fluxes together with the system states will be available to the entopreneur. Generally, the more detailed the model the simulation is based on, the more representative the results will be of what to expect in reality. Also, evidently, as the model is made more complex the computational effort required to run the simulation will increase. And, most importantly, any result obtained by means of modelling and simulation should always be compared to reality so that their validity can be verified.

\section{Applications of system modelling and simulation}

Modelling a proposed system and studying its anticipated dynamics by means of simulation can serve a number of purposes during project development. Having a reasonably good, dynamic model of the process available can also be very useful later, once the system has been built and is operational, when considering process modifications and improvements. Here only the issues of value in PPD are considered.

The first impetus concerns knowledge and data about the kinetics of the organism/feed combination that is being proposed for the project, together with a description of the environmental history to which it is planned to subject the larvae during their residence time in the rearing reactor. Thus, in order to construct a minimal kinetic model data is needed on the organism's growth curve and the various, related variables under the foreseen conditions. Depending on the type of process being planned, this corresponds to having available a limited version of Equations 1a and $1 \mathrm{~b}$ or $2 \mathrm{a}$ and $2 \mathrm{~b}$, specific to a narrow set of operating conditions. ${ }^{3}$ To create a more sophisticated kinetic model data will then be needed about the genetic and epigenetic diversity of the organism and what effect that has on the organism's growth curve as well as all the other, related variables under the proposed rearing conditions and any conditions that deviate from those. Essentially, every time another factor is taken into account in the model, a more complete version of the equations, or their equivalent, is required. To further expand the system model data on the age distribution of the eggs will also be needed. Hence, the decision to model the system and study its performance with simulation actually dictates the data requirement to make this possible and thus clarifies to the project planner what knowledge is essential for them to proceed in a deliberate and purposeful manner. Obviously, a fully developed kinetic model as expressed with Equation sets 1 or 2 that will yield an organism growth curve together with detailed data on all related variables as a function of organism age, genetic type, environmental history, time, etc., would be quite challenging to assemble, if only for the amount of experimental work required to obtain the data. As pointed out above it is, therefore, often more practical to initially start with a fairly simple model based on limited data and then expand the scope of the model gradually as data needs are identified. This principle applies to all the component models, i.e. of the competing organism, the disturbances, the control systems, etc., as well as that of the organism being reared.

The second purpose of modelling and simulating a system during PPD is probably the most important one: it is to facilitate and speed up the exploration of the possibility space for a project. In this sense, it is an extra tool for the project planner. Thus, the entopreneur might follow a traditional, evolutionary innovation path by starting off with a project concept and developing it by means of a combination of empirical and analytical means, learning from failure and success through a trial-and-

\footnotetext{
${ }^{3}$ Such information need not be available in analytical form; it can also be supplied in tabular format.
} 
error approach until it yields a satisfactory result, i.e. in this case, a combination of organism/feed, environmental conditions, etc. that works. This procedure can, however, be accelerated by having available a model of the system whose parameters can be changed at will so that one can, as it were, perform most of the trials in the virtual realm rather than the physical one. Generally, because it is much cheaper and faster to run simulations on a computer than to do experimental work with populations of organisms one can perform many more 'experiments' in this way. This means that the possibility space can be explored in a structured way in much greater detail than what is feasible with a strictly empirical approach so that it can be value mapped, giving the entopreneur more opportunity to locate any high-performance process operating areas and construct a path to an acceptable operating point, i.e. a combination of process type, organism characteristics, environmental conditions, etc. where the value of the process is acceptable.

Although there are many advantages to modelling and simulating a process, there are also some disadvantages. One disadvantage is that one needs to obtain sufficient data on the organism as well as the other system components to enable the construction of a reasonably representative model. This is partly offset by the gain of a good theoretical understanding of the whole system, and often a practical understanding as well. A second disadvantage is the effort and associated cost it takes to construct both the model and the simulation. Hopefully, that will be offset by a reduction in cost of the purely evolutionary, empirical approach because less experimental work will be needed to find a high-value process operating point. This also means the process development time will be shorter and the results will be better. The third main disadvantage of modelling and simulating is that one can easily become divorced from reality. This can and must be overcome by remaining critical of all simulation results and verifying all major conclusions empirically. In short, to obtain reliable results, during PPD a combination of the two approaches is necessary: experimental and simulation-based theoretical, the latter guiding the former and the former correcting the latter.

The dynamic modelling/simulation situation is actually not dissimilar to that dealt with in Part 1 of this set (Kok, 2021a) in which the overall heat and mass balances are estimated with a spreadsheet-based model whose parameters can be adjusted at will in order to investigate the process possibilities. The difference is that now the calculations are based on a dynamic model, but the intent is the same. In fact, considerable guidance on what parameter values to use for the simulation study will probably be derived from the overall mass and energy balance results. With simulation one can rapidly produce not only projected organism histories but also data on organism composition, overall process yield, by-product outputs, energy requirements, etc. for a large number of trial cases, i.e. for different control arrangements and for different environmental conditions that may be stable or that may vary with time, and that may be homogeneous or heterogeneous to some degree. In order to evaluate the success of a given set of trial parameters one usually combines the various process outputs into one or more objective functions which reflect the overall value of that combination. Basically, the objective functions will be a distillation of what the entopreneur wants to achieve with the project. For instance, the first objective function may result only in discrete values such as 0 and 1 , respectively indicating that the process is simply not viable at all (0) or at least physically possible (1), while the second one might represent financial reward value to the entopreneur. The results for $\mathrm{n}$ independent process variables can then be mapped in a space of $\mathrm{n}$ dimensions, the first objective function defining the possibility space for the process, within which value contours of the second objective function can be shown. This is illustrated in Figure 6 in which, for practical reasons, there are only two independent variables present. In this instance the possibility space is shown to be contiguous, but that is not necessarily always so. Also, only the region comprising the inner contours is considered 'of interest' as judged by the entopreneur, i.e. here the process performance is considered worthwhile. Because the number of independent variables may be substantial and resources limited, this type of map will often be based on fairly sparse data and will therefore be most suitable to guide the entopreneur on their search path through the possibility space to an acceptable process operating point. Evidently, one might accomplish the same with a purely empirical approach and, accordingly, two search paths are presented in Figure 6, one simulationguided and the other unguided. Here, the latter is shown as being longer and therefore requiring more effort while also ending up at a somewhat lower valued operating point for the process, although still well within the region of interest. Reality being capricious as it is, there is no guarantee that a search will always unfold in this manner but what is illustrated here corresponds to the major purpose of the entire modelling and simulation exercise: to shorten the search path and to arrive at a higher value operating point for the process than with an unguided approach.

The third purpose of modelling a process during PPD and simulating its performance is to allow the entopreneur to engage in reasoning that is somewhat more speculative. Thus, once a reasonably representative value contour map has been established and a feasible, high-value operating point for the process has been identified the entopreneur may start to wonder what other factors could possibly be altered so as to improve the situation to a yet-higher value point. For instance, could a different feed be found or could the feed composition be adjusted? Might varying the temperature during larval growth have a positive influence? Referring to Figure 4 in which the organism reject rates 


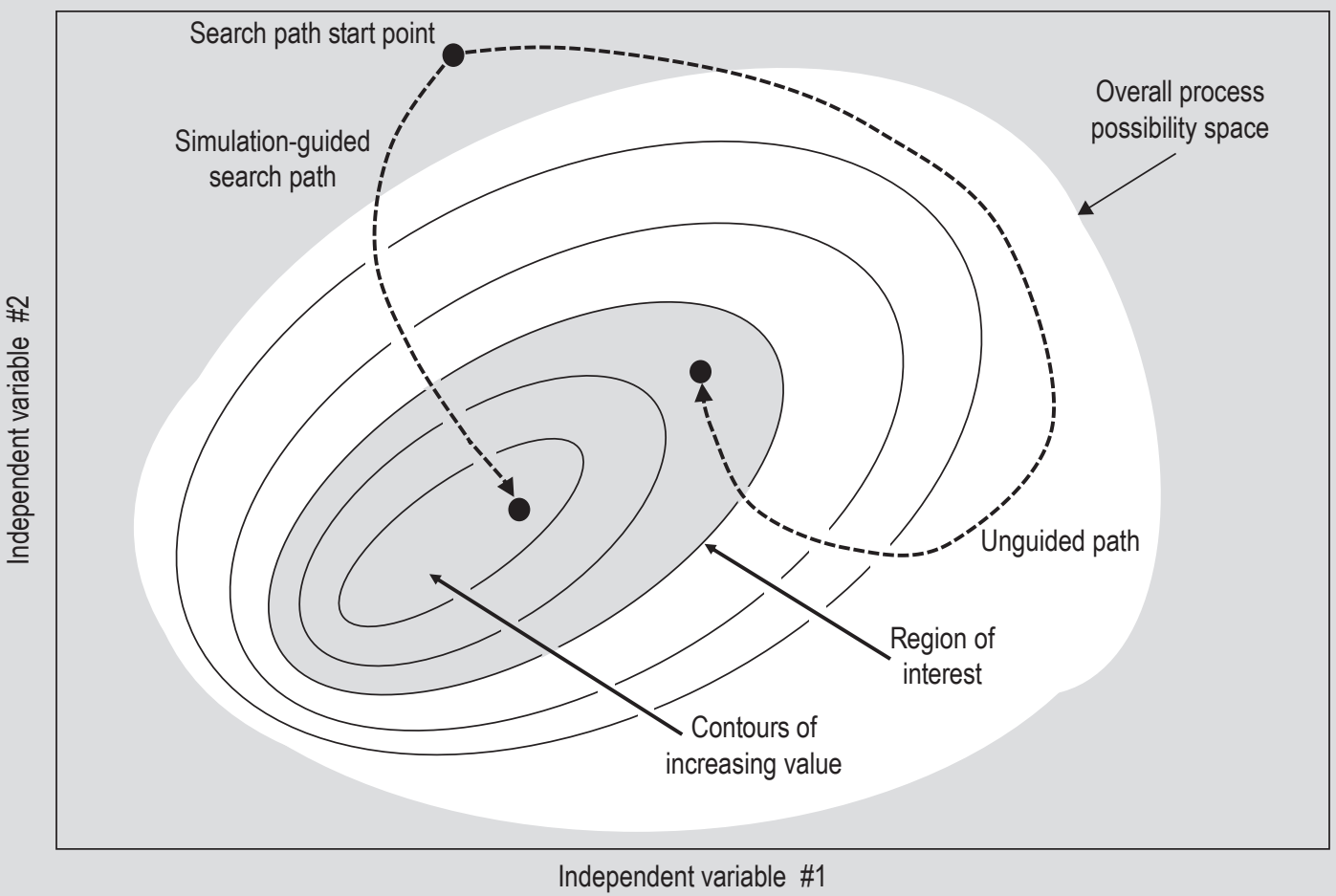

Figure 6. Value contours and development paths.

for Cases 3 and 4 are fairly high due to diversity, nonsynchronicity and environmental heterogeneity questions would naturally arise about the feasibility of breeding a genetically less diverse strain, obtaining eggs with a narrower age distribution, arranging the reactor so that the environment will be less heterogeneous, etc. The potential impacts of any such possible adjustments can often be initially gauged with the existent models and data although, in order to address the queries in depth additional data may be needed. Thus, a speculative approach may lead one to identify and specify new data requirements which may then need to be fulfilled through more experimental work. This would be the case if, for example, different feeds were to be considered or if the option of varying the temperature during larval rearing were to be contemplated. Generally, in more speculative reasoning any of the system components, conditions or parameters can be addressed and adjusted including the models, the minimal requirements imposed by the entopreneur, the form of the objective functions and even the process type itself. In this flexibility the utility of simulation is manifested most clearly as a formal, structured approach to reasoning about a project and its associated possibility space. It should be noted here that, with fundamental aspects of the project being changed as part of the speculations, the possibility space and its value contours can undergo fairly dramatic changes in both shape and location, and even in dimension. Evidently, what might be possible under one set of restraints and goals might not be possible under another and vice versa.
The fourth purpose of utilising a simulation approach during PPD is to gain insight into the sensitivity of the process to two main sources of instability: variation in any of the main variables such as feed composition, genetic characteristics of the organism, a change in the competing organisms, etc. and disturbances such as short-term fluctuations in cooling air temperature or temperature distribution. Developing an understanding of the system's sensitivity to any of these factors and combinations of them facilitates the development of design specifications for the inputs, the system itself as well as the performance of the control system. For instance, a system's performance at a high-value operating point in the possibility space might turn out to be quite sensitive to the age distribution of the eggs, leading directly to the question whether it is feasible to obtain eggs with a very narrow age distribution - a specification for an input. Similarly, it may be sensitive at that point to genetic diversity, leading to the question whether it is possible to obtain eggs that are less dissimilar - another input specification. Larval development may be quite sensitive to environmental conditions during rearing so that the development distribution at harvest will depend heavily on constancy and homogeneity of those conditions - this would lead to specifications for the reactor and the control system, e.g. how far the bed temperature can be allowed to deviate from its set point. As well, the posing of specific questions in response to gaining an understanding of sensitivities will lead to the formulation of more general queries about the system as a whole. These might be related to the feasibility 
and desirability of a breeding program, the design and development of sensors and other control components specifically for applications in entotechnology, the creation of artificially intelligent components to operate the process optimally so as enforce synchronisation, etc.

In summary, the overall motivation for using a simulation approach during PPD is to examine a larger possibility space for the project than is normally feasible with a purely experimental approach and to arrive at an acceptable operating point for the process. At the same time the entopreneur will gain considerable insight into anticipated design specifications for the process and its equipment as well as for the inputs and the control system. This will allow them to estimate what effort will be required and to assess the feasibility of the plan that is in place. As a first possibility it is not inconceivable that the initial search through the possibility space will lead to a process operating point well within the region of interest while the process at this point is not overly sensitive to variations and disturbances and, upon first examination, is also fairly easy to implement with off-the-shelf equipment. This is an unusually fortunate situation and one would probably proceed right away with experimental work to ascertain it is indeed true, attempt to improve the situation further through some more simulation work, and then proceed with the next project development step. As a second possibility, it is also quite feasible that within the possibility space which is initially established there lies a sub-space that appears promising but whose promise is contingent on a somewhat better definition of the situation, i.e. a more detailed description of the that region of the possibility space. This represents a data requirement and one would proceed in this case with obtaining those data on, for example, egg age distribution or an aspect of the kinetic model, and then compute and map a modified possibility space with new simulation runs. It is actually not unlikely that in reality this will happen a number of times so that during the simulation study the following sequence will be repeated: examination of the possibility space; path searching to a likely process operating point or region of interest (Figure 6); scrutiny and assessment of the system performance at this operating point; composition of questions and consideration of alternatives; setting further data requirements; fulfilling data requirements; compute and map new possibility space, etc. It should be taken into account, as a result of new data entering the system, the possibility space for the process can shift and change shape considerably.

What may also happen is that examination of the possibility space leads to doubts about the viability of the project as put forward. Thus, with the organism/feed combination and under the conditions that have been proposed, even after exploring a number of alternatives, there simply is no region of interest or, in extreme cases, not even a possibility space. Although this may be disappointing to the entopreneur planning the project, because much wasted effort and investment will be avoided, it is really a boon. At this point the entopreneur can either abandon the project altogether or engage in a much wider-ranging exploration of feeds, organisms, process types, by-products, etc., which will then lead to efforts to breed new varieties of organisms, to develop equipment, to innovate novel process types, and to engage in further simulation study based on those.

One issue that bears repeating at this point is that, whereas in the above almost all reference is to simulation performed in a formal manner, i.e. with mathematical and data-based models on computer platforms, it is also possible to do all this in an informal manner, i.e. with thought processes running on human brains. Some advantages of the latter approach are that brains are far superior in terms of applying intuitive reasoning to detect very complex patterns and to be creative so that it is more likely that far-flung prospects will be included in the considerations of alternatives. On the other hand, computer-based reasoning is often more consistent and repeatable so that computational results are usually easier to explain and justify. In many cases a combination of the two approaches will be fruitful.

\section{Discussion and conclusions}

The main point of this paper is: before an entopreneur commits to a specific plan for a project it's a good idea to think in detail about the dynamics of the system as well as the overall heat and mass balances. A modelling and simulation approach based on experimental data allows for the integration of knowledge about organism kinetics, reactor configuration, process performance and control system activity so that the system dynamics can predicted. This facilitates the rational analysis of possibilities for a project and the examination of alternatives.

At the conclusion of a project's modelling/simulation phase the aspiring entopreneur should have a fair understanding of all quantitative aspects of the project and the selected process type, including heat and mass flows, kinetics, inputs and outputs, dynamics, equipment requirements, etc. Some aspects of the various process types, equipment and operating schemes will be dealt with in Part 3 of this set (Kok, 2021b).

\section{Conflict of interest}

The author declares no conflict of interest.

\section{References}

Aiba, S., Humphrey, A.E. and Millis, N.F., 1973. Biochemical engineering ( $2^{\text {nd }} E d$.). Academic Press, Cambridge, MA, USA, 434 pp. 
Diener, S., Zurbrugg, C., Gutierrez, F.R., Nguyen, D.H., Morel, A., Koottatep, T., Tockner, K., 2011. Black soldier fly larvae for organic waste treatment - prospects and constraints. In: Alamgir, M., Bari, Q.H., Rafizul, I.M., Islam, S.M.T., Sarkar, G., Howlader, M.K. (eds.) Proceedings of the WasteSafe 2011 - $2^{\text {nd }}$ International Conference on Solid Waste Management in the Developing Countries, February 13-15, 2011, Khulna, Bangladesh, pp. 1-8.

Kok, R., 2017. Insect production and facility design. In: Van Huis, A. and Tomberlin, J.K. (eds.) Insects as food and feed: from production to consumption. Wageningen Academic Publishers, Wageningen, the Netherlands, pp. 142-172.

Kok, R., 2021a. Preliminary project design for insect production: part 1 - overall mass and energy/heat balances. Journal of Insects as Food and Feed 7: 499-509. https://doi.org/10.3920/JIFF2020.0055

Kok, R., 2021b. Preliminary project design for insect production: part 3 - sub-process types and reactors. Journal of Insects as Food and Feed 7: 525-539. https://doi.org/10.3920/JIFF2020.0145
Kok, R., 2021c. Preliminary project design for insect production: part 4 - facility considerations. Journal of Insects as Food and Feed 7: 541-551.https://doi.org/10.3920/JIFF2020.0164

Nyakeri, E.M., Ogola, H.J.O., Ayieko, M.A. and Amimo, F.A., 2017. Valorisation of organic waste material: growth performance of wild black soldier fly larvae (Hermetia illucens) reared on different organic wastes. Journal of Insects as Food and Feed 3: 193-202. https://doi.org/10.3920/JIFF2017.0004

Parrott, L. and Kok, R., 2001a. Use of an object-based model to represent complex features of ecosystems. In: Minai, A.A. and Bar-Yam, Y. (eds.) Unifying themes in complex systems. Springer, Berlin, Heidelberg, Germany.

Parrott, L. and Kok, R., 2001b. A generic primary producer model for use in ecosystem simulation. Ecological Modelling 138: 75-99.

Parrott, L. and Kok, R., 2002. A generic, individual-based approach to modelling higher trophic levels in simulation of terrestrial ecosystems. Ecological Modelling 154: 151-178. 
